\title{
Stability charts of the slope with tensile strength cut-off
}

\author{
Yi He ${ }^{\text {i) }}$ and Yan Liu ${ }^{\text {ii) }}$
}

i) Associate Professor, Faculty of Geosciences and Environmental Engineering, Southwest Jiaotong University, Chengdu 611756, China. ii) Ph.D student, Department of Civil and Structural Engineering, Kyushu University, Fukuoka819-0395,Japan.

\begin{abstract}
The tensile strength of the soil predicted by the classical Mohr-Coulomb (M-C) yield criterion is suspicious, because it is determined from the extrapolation of the test results in the compressive regime. In this case, the classical M-C yield criterion is modified by reducing the tensile strength in the tensile regime. An analytical approach under the framework of limit analysis is carried out incorporating with the modified yield criterion to obtain the upper-bound solutions of the slope stability. Stability charts for slopes with tensile strength cut-off under different seismic accelerations are provided. It is revealed that considering the effects of tension cut-off, the stability factor is reduced by $55 \%$ for vertical slopes subjected to seismic loads.
\end{abstract}

Keywords: slopes; seismic; tension cut-off; stability chart

\section{INTRODUCTION}

The linear Mohr-Coulomb (M-C) yield criterion, as shown in Fig. 1(a), are typically employed in the analysis of slope stability. However, testing for material properties, is typically carried out in the compressive regime, the tensile strength is then burdened by uncertainties, as it is a result of extrapolation of test results into the tensile regime (Michalowski 2017). That is why for some geomaterials, especially the brittle materials, the strength is usually described by the nonlinear yield criterion, and the tensile strength is adjusted depending on the state of the material (Park et al. 2017). Drucker and Prager (1952) modified the M-C criterion by eliminating the tension, as shown in Fig. 1(c), to explain the discrepancy between the lower and upper results of the critical height of the vertical bank. In addition, they suggested that "...the concept of a soil unable to take tension clearly requires much additional study."

It is suggested by Duncan and Wright (2005) that, if the tensile stress is considered in slope analyses, it should be either eliminated by introducing a tension crack or reduced by truncating the yield envelope. The former approach has been extensively studied with various methods (Utili 2013; He et al. 2019), and the later has been introduced as the tensile strength cut-off. Michalowski (2017) adopted the concept of tension cutoff, and analyzed the stability of intact slopes under static conditions, among others (Drucker \& Prager 1952; Abd et al. 2017). These researchers mainly studied the slope problems under static conditions. However, systematic analyses of seismic slopes have not been fully

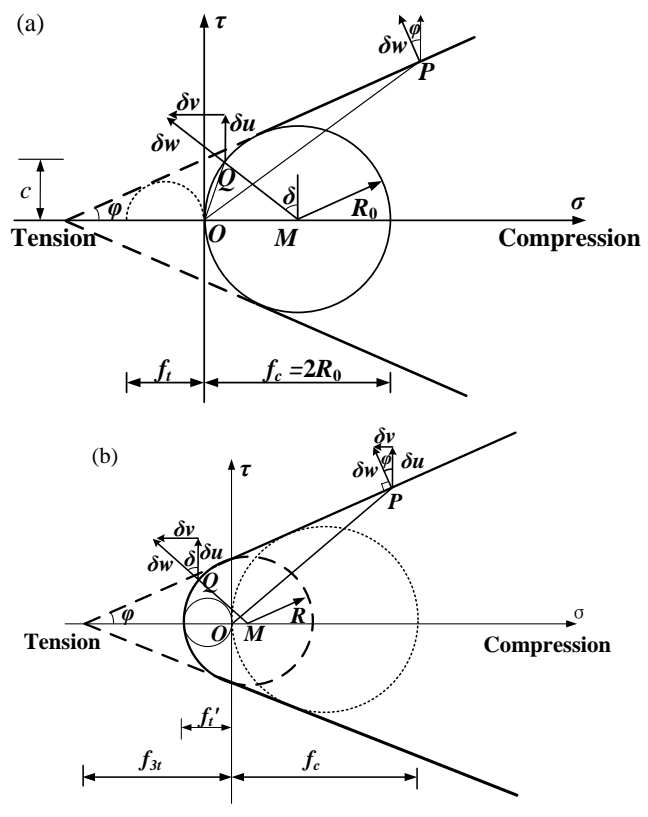

(c)

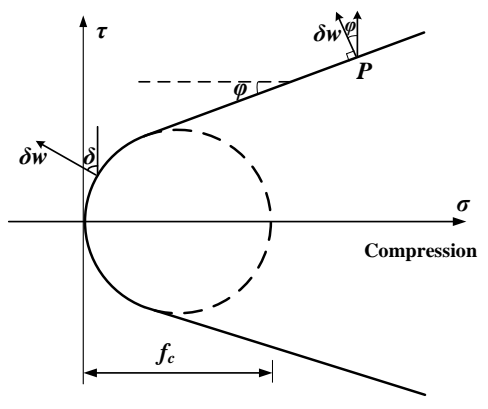

Fig. 1. (a) Classical M-C criterion (the tensile regime is depicted by dashed lines), (b) yield criterion with partial tension cut-off, and (c) yield criterion with zero tensile strength (after Chen 1975). 
provided.

In this paper, the reduction of the tensile strength of the cemented soil is discussed. Considering the effects of the tensile strength coefficient $\xi$ (which is described in the next section), the stability number and safety factor of the slopes are studied. The difference between the results obtained by the conventional $\mathrm{M}-\mathrm{C}$ yield criterion and the tension cut-off model is also discussed.

\section{TENSILE STRENGTH CUT-OFF}

As mentioned previously, the tensile strength of soil in the $\mathrm{M}-\mathrm{C}$ yield criterion is not obtained by routine testing, but by extrapolation from the compressive regime to the tensile regime. As shown in Fig. 1(a) and (b), the uniaxial tensile strength $f_{\mathrm{t}}$ and triaxial tension $f_{3 \mathrm{t}}$ in the conventional $\mathrm{M}-\mathrm{C}$ yield criterion are $2 c \cos \varphi /(1+$ $\sin \varphi)$ and $c / \tan \varphi$, respectively. Chen (1975) modified the $\mathrm{M}-\mathrm{C}$ criterion by eliminating the tension, as shown in Fig. 1(b) and (c), to estimate the bearing capacity of brittle materials, e.g., concrete blocks or rock. Park et al. (2017) focused on the tensile strength of bonded soils, and studied the static soil slope stability by considering the partial cut-off with uniaxial tensile strength. They demonstrated that decreasing the tensile strength from the critical condition reduces the stability factor, especially for steep slopes. However, under dynamic conditions, the effect of the tension cut-off, if any, on the slope stability has not been sufficiently studied. This paper proposes a kinematic approach using limit analysis under seismic loading and presents stability charts. For cemented soils, the conventional $\mathrm{M}-\mathrm{C}$ yield criterion is modified by introducing the tension cut-off. Further details on the yield function of the tensile strength cutoff and the analysis of the energy dissipation rate for the modified criterion can be found in references (He et al. 2019).

\section{PSEUDO-STATIC APPROACH}

Because of the presence of seismic forces, two types of external work are considered: the rate of external work due to the soil weight, $W_{\mathrm{r}}$ (Michalowski, 2017); and the rate of external work due to the seismic load, $W_{\mathrm{s}}$, i.e.,

$$
W^{s}=W_{A D B O A}^{s}-W_{A C D}^{s}
$$

where $W_{A D B O A}^{s}$ and $W_{A C D}^{s}$ are the work rates of the corresponding blocks, i.e., ADBOA and ACD respectively shown in Fig. 2, induced by seismic loads. $W_{A D B O A}^{s}$ and $W_{A C D}^{s}$ are given in the literature (e.g., Chen Utili \& Abd 2016; He et al. 2019).

As illustrated in Fig. 2, if the conventional M-C yield criterion is applied, the failure surface in the homogeneous slope is log-spiral (the shear band ADB in Fig. 2). When the tension cut-off is considered, the failure surface more likely follows the shape of curve $\mathrm{CDB}$ in Fig. 2. Along the failure curve CDB, the rate of internal dissipation $W_{\mathrm{d}}$ consists of two components:

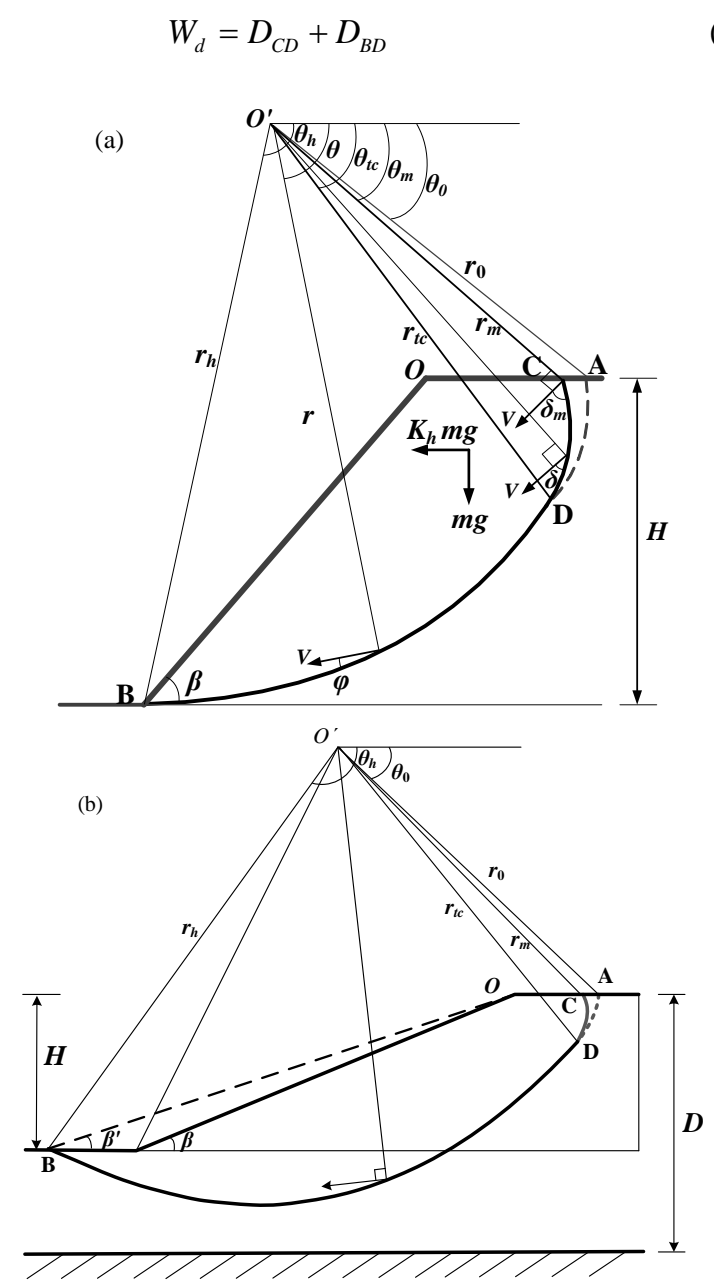

Fig. 2. Failure mechanism for slopes with tensile strength cut-off: (a) toe failure mechanism and (b) base failure mechanism (after Michalowski 2017).

where $D_{\mathrm{CD}}$ and $D_{\mathrm{BD}}$ are the rate of dissipation along failure curves $\mathrm{CD}$ and $\mathrm{DB}$. The equations for $D_{\mathrm{CD}}$ and $D_{\text {DB }}$ are adopted from Michalowski (2017).

The upper bound is derived by imposing an energy balance for the failing wedge OBDC, and the stability factor is calculated as follows:

$$
N_{\xi}=\frac{\gamma H}{c}=\frac{H}{r_{0}} \frac{\left(D_{C D}+D_{D B}\right) / c \omega r_{0}^{2}}{S_{a}+K_{h} D_{y}}
$$

where $S_{\mathrm{a}}=f_{1}-f_{2}-f_{3}-f_{4}-t_{1}+t_{2}+t_{3}$,

$$
D_{y}=f_{1}^{s}-f_{2}^{s}-f_{3}^{s}-f_{4}^{s}-t_{1}^{s}+t_{2}^{s}+t_{3}^{s}
$$

and the parameters $H / r_{0}, f_{\mathrm{i}}-f_{4}, t_{1}-t_{3}, f_{1}{ }^{\mathrm{s}}-f_{4}{ }^{\mathrm{s}}, t_{1}{ }^{\mathrm{s}}-t_{3}{ }^{\mathrm{s}}$ can be found in literature (He et al. 2019), and $N_{\xi}$ is the stability factor (it can be used, in practice, as an efficient tool and is routinely used as a quick reference for preliminary assessment of slope safety). The subscript $\xi$ varies from 0 to 1 ( $\xi=1$ for the conventional $\mathrm{M}-\mathrm{C}$ yield condition; $0<\xi<1$ for a partial tensile strength cut-off, and $\xi=0$ for a full tension cut-off).

As indicated by Michalowski (2002), when the slopes with small values of slope angle $\beta$ and internal friction angle $\varphi$ are subjected to stronger seismic excitation, the dimensions of the most adverse failure mechanism tend 
to infinity. In order to obtain a more rational value of $N_{\xi}$, the depth of the failure mechanism is limited to a realistic value $D$ below the crest of the slope (Fig. 2(b)). In this paper the depth factor $\mathrm{D} / \mathrm{H}=2$ is adopted for slopes subjected to seismic excitation. The independent variables in the above equation are $\theta_{0}, \theta_{\mathrm{m}}, \theta_{\mathrm{tc}}$, and $\theta_{\mathrm{h}}$. The critical value, $N_{\xi}=\gamma H / c$, is sought using a combination of the above variables; further, the dependent variable $\delta_{\mathrm{m}}$ is a function of $\theta_{0}, \theta_{\mathrm{m}}, \theta_{\mathrm{tc}}$.

In addition, considering the modified criterion with reduced tensile strength, the stability charts for slopes subjected to seismic loading are presented. Although computational tools have made most graphical methods and charts obsolete, stability charts for slopes are still routinely used in practice. The charts presented here are based on the kinematic approach of limit analysis, which leads to an upper bound on the safety factor $F_{\mathrm{s}}$. The safety factor is defined as

$$
\left\{\begin{array}{l}
c_{f}=c / F_{s} \\
\varphi_{f}=\arctan \left(\tan \varphi / F_{s}\right)
\end{array}\right.
$$

where $c_{f}$ and $\varphi_{f}$ are the soil strength parameters necessary only to maintain the structure in limit equilibrium and are sometimes referred to as the mobilized strength parameters (Michalowski 2002; Zhao et al. 2015). By substituting Eq. (4) into Eq. (3) and rearranging, the safety factor is expressed as:

$$
F_{S}=\frac{c}{\gamma H} \frac{\left(D_{C D}+D_{D B}\right) / c \omega r_{0}^{2}}{S_{a}+K_{h} D_{y}} \frac{H}{r_{0}}
$$

This is an implicit form of $F_{s}$. For convenience in calculating $F_{\mathrm{s}}$, the stability charts are presented here for slopes with given $c / \gamma H, \beta, \varphi$, and $K_{\mathrm{h}}$ values.

The sequence quadratic program (SQP) is used to find the upper bound results. The accuracy of the SQP depends on the incipient inputs of the variants. The random trials method is used to estimate the global minimum. Then the corresponding variant values are taken as the incipient inputs, and finally a minimization procedure is performed using SQP. The upper bound results obtained by this optimization technique under static conditions are validated using data obtained from the published literature.

\section{STABILITY FACTOR FOR SLOPES WITH REDUCED TENSILE STRENGTH SUBJECTED TO SEISMIC LOADING}

The proposed approach is numerically validated using the results presented by Michalowski (2017), as shown in Table 1. The results obtained in this paper are close to those obtained by Michalowski (2017), highlighting the suitability of the optimization procedures.

A parametric study is performed to investigate the effects of the tensile strength of soils on the slope stability, under both static and dynamic conditions. When the tension cut-off is taken into account, the least
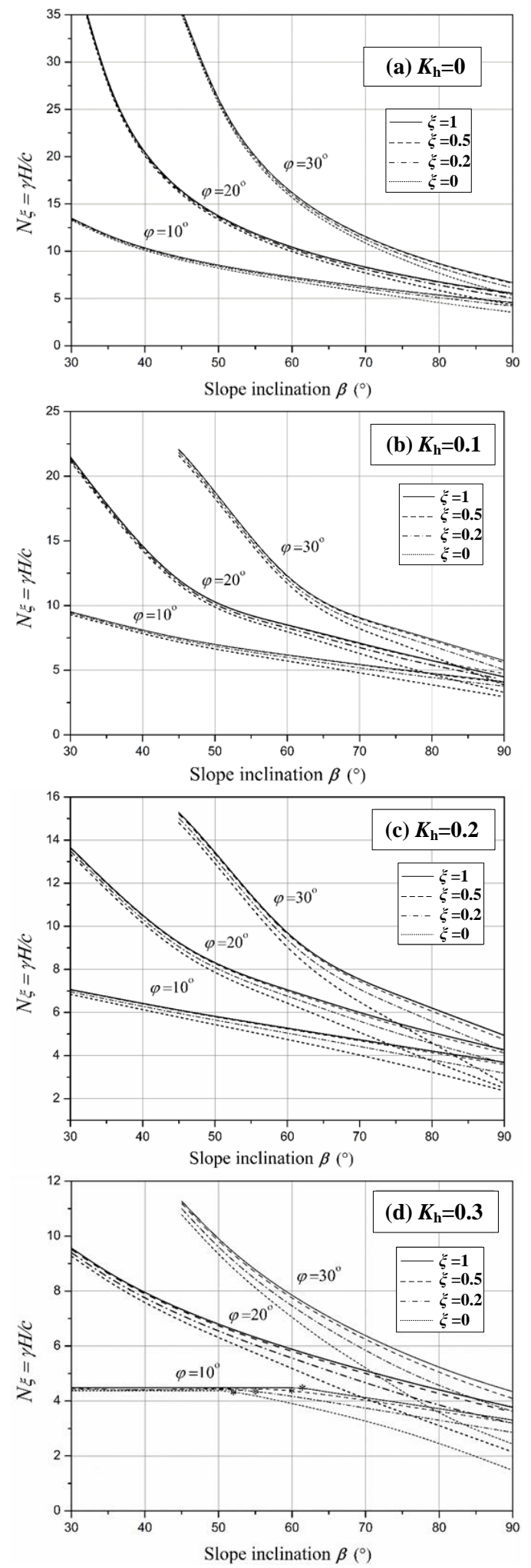

Fig. 3. Stability factor $(\gamma H / c)$ for slopes with tensile strength cutoff under earthquake loading; (a): $K_{\mathrm{h}}=0$, (b): $K_{\mathrm{h}}=0.1$, (c): $K_{\mathrm{h}}=$ 0.2 , and (d): $K_{\mathrm{h}}=0.3$.

upper bound of the stability factor is obtained by searching for the minimum of $N_{\xi}$ in Eq. (3). The obtained upper bounds are plotted in Fig. 3 against the inclination angle $\beta$ for $\varphi=10^{\circ}, 20^{\circ}$, and $30^{\circ}$, where $K_{\mathrm{h}}$ ranges from 0 to 0.3 , and $\xi$ is varied from 0 to 1 . Fig. 3 shows that the 
slope stability decreases with decreasing $\xi$ under both static and dynamic conditions. Increases in the inclination angle $\beta$ and horizontal seismic coefficient $K_{\mathrm{h}}$ result in more significant reductions in slope stability. As shown in Fig.3 (d), for $\varphi=20^{\circ}$ and $30^{\circ}$, the toe failure mechanism occurs. However for $\varphi=10^{\circ}$, the toe failure mechanism occurs after the slope angle reaches the marked value, before which the base failure mechanism is observed.

Table 1 Validation of the stability factor $\gamma H / c$.

\begin{tabular}{|c|c|c|c|c|c|}
\hline \multirow{3}{*}{$\begin{array}{c}\text { Slope } \\
\text { angle } \\
\beta\end{array}$} & \multirow{3}{*}{$\xi$} & \multicolumn{4}{|c|}{ Internal friction angle $\varphi$} \\
\hline & & \multicolumn{2}{|c|}{$10^{\circ}$} & \multicolumn{2}{|c|}{$30^{\circ}$} \\
\hline & & $\begin{array}{c}\text { Reference } \\
\text { [6] }\end{array}$ & $\begin{array}{l}\text { Present } \\
\text { method }\end{array}$ & $\begin{array}{c}\text { Reference } \\
{[6]}\end{array}$ & $\begin{array}{l}\text { Present } \\
\text { method }\end{array}$ \\
\hline \multirow{4}{*}{$30^{\circ}$} & 0 & 13.26 & 13.268 & & \\
\hline & 0.2 & 13.39 & 13.397 & & \\
\hline & 0.5 & 13.48 & 13.485 & & \\
\hline & 1 & 13.49 & 13.499 & & \\
\hline \multirow{4}{*}{$60^{\circ}$} & 0 & 6.84 & 6.852 & 15.49 & 15.499 \\
\hline & 0.2 & 7.1 & 7.104 & 15.77 & 15.776 \\
\hline & 0.5 & 7.24 & 7.245 & 15.99 & 15.987 \\
\hline & 1 & 7.25 & 7.258 & 16.03 & 16.035 \\
\hline \multirow{4}{*}{$90^{\circ}$} & 0 & 3.47 & 3.486 & 5.13 & 5.132 \\
\hline & 0.2 & 4.2 & 4.190 & 6.08 & 6.079 \\
\hline & 0.5 & 4.54 & 4.538 & 6.59 & 6.585 \\
\hline & 1 & 4.58 & 4.582 & 6.68 & 6.686 \\
\hline
\end{tabular}
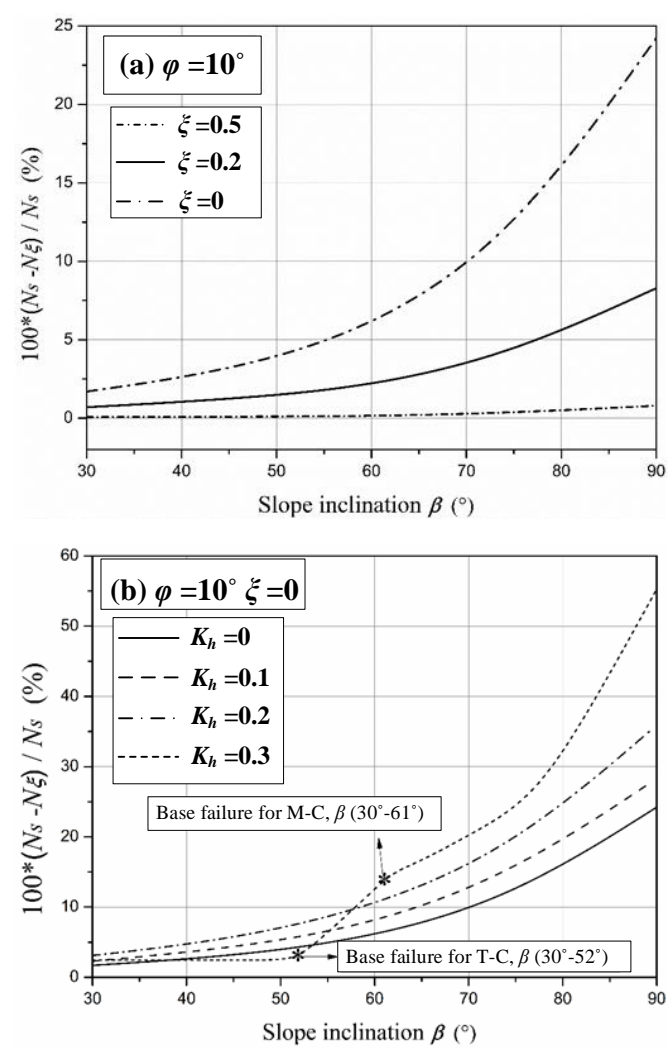

Fig. 4. Reduction of stability factor for (a) static condition with $\varphi$ $=10^{\circ}$ and (b) dynamic condition with $\varphi=10^{\circ}$ and $\xi=0$.

Another interesting finding is that the stability factor decreases rapidly with decreasing $\varphi$. To quantify the reduction in the stability factor induced by seismic
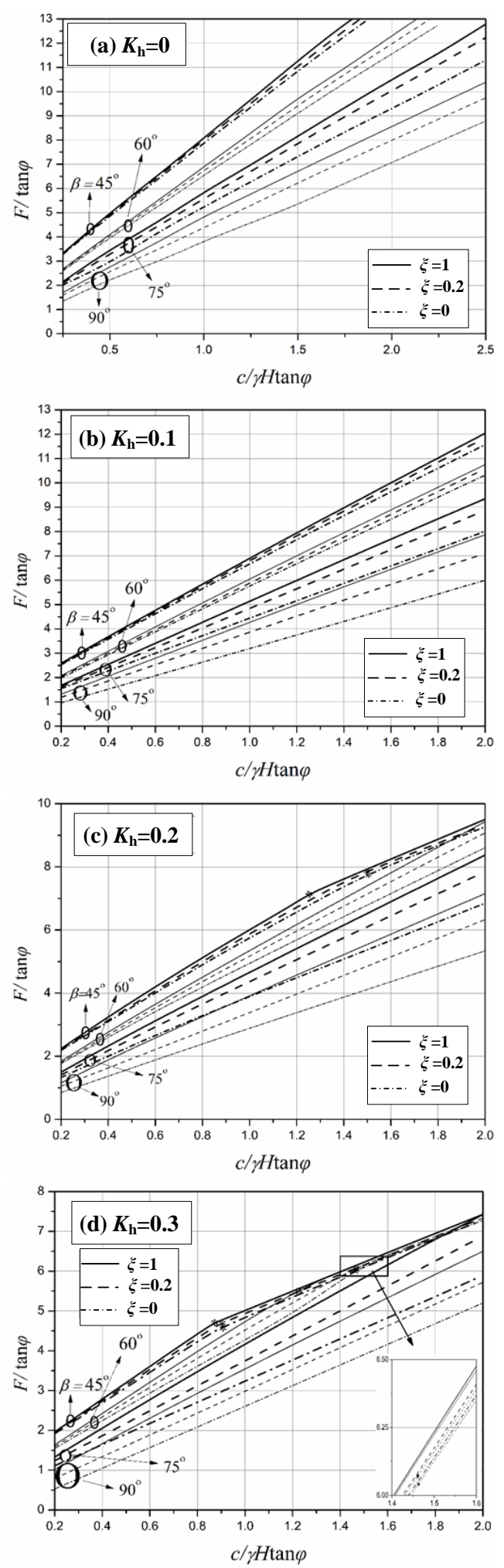

Fig. 5. Stability charts for slopes with tensile strength cut-off under earthquake loading, (a): $K_{\mathrm{h}}=0$, (b): $K_{\mathrm{h}}=0.1$, (c): $K_{\mathrm{h}}=0.2$, and (d): $K_{\mathrm{h}}=0.3$.

excitation and truncation of the tensile strength, the following equation is employed:

$$
P=\left|N_{s}-N_{\xi}\right| / N_{s} \times 100 \%
$$

where $\mathrm{P}$ represents the percentage of reduction in the 
stability factor, and Ns is the slope stability factor for the conventional $\mathrm{M}-\mathrm{C}$ yield criterion. The subscript $\xi$ in the first term on the right-hand side ranges from 0 to 1 .

The results of Eq. (6) are presented graphically in Fig. 4. As shown in Fig. 4(a), if $\xi$ is larger than 0.5 when subjected to static conditions, the influence of the tension cut-off is negligible. However, if the tensile strength is fully cut off (i.e., $\xi=0$ ), the reduction in the stability factor is significant, even under static conditions (for a vertical slope, the reduction is roughly $25 \%$ ). The effects of the two parameters (the seismic coefficient $K_{\mathrm{h}}$ and slope angle $\beta$ ) on the reduction in the slope stability factor under dynamic conditions are illustrated in Fig. 4(b). For $\varphi=10^{\circ}, \xi=0$, and $K_{\mathrm{h}}=0.3$, Ns is reduced by approximately $2.5 \%$ to $55 \%$ as the slope angle increases from $30^{\circ}$ to $90^{\circ}$. Fig. 4(a) and (b) show that the influence of the tension cut-off is obvious for steep slopes, and when a seismic load is applied, this influence is larger. In summary, Fig. 4(b) suggests that under dynamic conditions, it is conservative to consider the tensile strength cut-off when analyzing the slope stability.

\section{STABILITY CHARTS FOR SLOPES WITH REDUCED TENSILE STRENGTH SUBJECTED TO SEISMIC LOADING}

Fig. 5 shows the safety factors of slopes subjected to different seismic loads considering the tension cut-off effect. When the classical M-C yield criterion is recovered with $\xi=1$, the calculated results of safety factors agree well with Michalowski (2002). Fig. 5(c) and 5(d) demonstrate toe failure mechanisms, except for the cases in Fig. 5(c) $\left(\beta=45^{\circ}\right)$ and in Fig. $5(\mathrm{~d})\left(\beta=45^{\circ}\right.$ and $\beta=60^{\circ}$ ). For these cases, the base failure mechanism occurs after the value of $c / \gamma H \tan \varphi$ reaches the marked value, before which the toe failure mechanism is observed.

\section{CONCLUSIONS}

The kinematic approach of limit analysis under seismic loading was proposed, and a comprehensive parametric analysis was conducted to estimate the influence of the tensile strength cut-off on the stability factor and permanent displacement of a seismic slope. To obtain the least upper bound results, the SQP optimization procedure was used. The proposed approach was numerically validated with literature data. Two seismic stability indicators, i.e., the stability factor, and safety factor were studied. All results were presented in graphical forms.

The stability factor decreased with decreasing $\xi$. The steep slopes subjected to seismic loading are most vulnerable to tension cut-off. For example, for vertical slopes with a full tension cut-off, if the horizontal seismic coefficient was large (e.g., $K_{\mathrm{h}}=0.3$ ), the reduction in the stability factor reached nearly $55 \%$. In addition, stability charts were presented as a reference to assess the slope safety.

\section{ACKNOWLEDGEMENTS}

This work was supported by the National Natural Science Foundation of China (Grant Nos. 51608454 and 51609204).

\section{REFERENCES}

1) Abd A., Utili S. (2017): Design of geosynthetic-reinforced slopes in cohesive backfills. Geotextiles and Geomembranes 45, 627-641.

2) Chen W. F. (1975): Limit Analysis and Soil Plasticity. Amsterdam, Netherlands: Elsevier Science.

3) Drucker D. C., Prager W. (1952): Soil mechanics and plastic analysis or limit design. Quarterly of Applied Mathematics, 10(2):157-165.

4) Duncan J. M., Wright S. G. (2005): Soil Strength and Slope Stability. Hoboken, NJ: John Wiley and Sons.

5) He Y, Liu Y, Zhang Y B, Yuan R. Stability assessment of three-dimensional slopes with cracks. Engineering Geology 2019; 252: 136-144.

6) Michalowski R. L. (2017): Stability of intact slopes with tensile strength cut-off. Geotechnique, 67(8):1-8.

7) Michalowski R L. (2002): Stability charts for uniform slopes. Journal of Geotechnical \& Geoenvironmental Engineering, 128(4):351-355.

8) Park D., Michalowski R. L. (2017): Three-dimensional stability analysis of slopes in hard soil/soft rock with tensile strength cut-off. Engineering Geology, 229: 73-84.

9) Utili S. (2013): Investigation by limit analysis on the stability of slopes with cracks. Geotechnique, 63(2):140-154.

10) Zhao L. H., Yang F., Zhang Y., et al. (2015): Effects of shear strength reduction strategies on safety factor of homogeneous slope based on a general nonlinear failure criterion. Computers \& Geotechnics, 63:215-228. 